\section{Perfume Microencapsulation by Complex Coacervation}

\author{
Andreas Meyer*
}

Abstract. Many techniques are known for the microencapsulation of substances. Complex coacervation is one of them. Generally, gelatin and gum arabic are used to form the capsule wall. Gum arabic may be replaced by other colloidal polymers such as pectin. Most often, pure substances are microencapsulated, but microencapsulation of complex mixtures such as perfumes is also possible. This paper reports on microencapsulation of perfumes using complex coacervation. The influence of the wall material on the microcapsule characteristics is given. croencapsulated perfume is protected from evaporation and maintains its original composition, as there is no preferential evaporation of its more volatile components. The performance of these microcapsules is compared to microcapsules made from gelatin and gum arabic.

\section{Experimental}

Perfume was microencapsulated using the technique of complex coacervation with gelatin and pectin as capsule wall forming materials. A 1-l automated laboratory reactor (Contalab, Mettler-Toledo $A G$ ) was used for the experiments. The process scheme is given by Fig. I. First, 75 $\mathrm{g}$ of gelatin and $15 \mathrm{~g}$ of pectin were dissolved in $2500 \mathrm{~g}$ of $\mathrm{H}_{2} \mathrm{O}$ at $50-55^{\circ} .460 \mathrm{~g}$ of perfume were added and dispersed by stirring. The stirring speed is adjusted to the desired microcapsule size. Then, $2.5 \mathrm{~g}$ of AcOH were added to form the complex coacervate, a sticky substance made from gelatin and pectin. The mixture was slowly cooled to $10^{\circ}$. During cooling, the complex coacervate stuck to the perfume droplets, thus form-

\section{Introduction}

Microencapsulation of solids and liquids is a convenient method to shield substances from the environment. In general, the purpose is to facilitate the handling or the application of the substance and to protect the substance from the environment. Thus, a bitter drug will be swallowed without repugnance as microencapsulation permits masking of the taste. A sticky substance may be converted into a free flowing powder by microencapsulation techniques. A liquid can be blended with solids when microencapsulated.

Many techniques are available for microencapsulation. Extensive reviews were made by Sparks [1] and Gutcho [2], and the state of the art was recently summarized [3] . If a substance is to be encapsulated by complex coacervation its solubility in water has to be small.

Usually, gelatin and gum arabic are used to form the capsule wall, although other colloids can also be used. In the review of Gutcho [2], pectin is mentioned. De Leeuw [4], Nakagawa et al. [5], and Michael [6] report on microencapsulation of perfumes using complex coacervation and preferentially gum arabic and gelatin to form the capsule wall. This work reports on perfume microencapsulation by complex coacervation using gelatin and pectin as capsule wall materials. The mi-

*Correspondence: Dr. A. Meyer

Givaudan-Roure $S A$

$\mathrm{CH}-1214$ Vernier-Genève

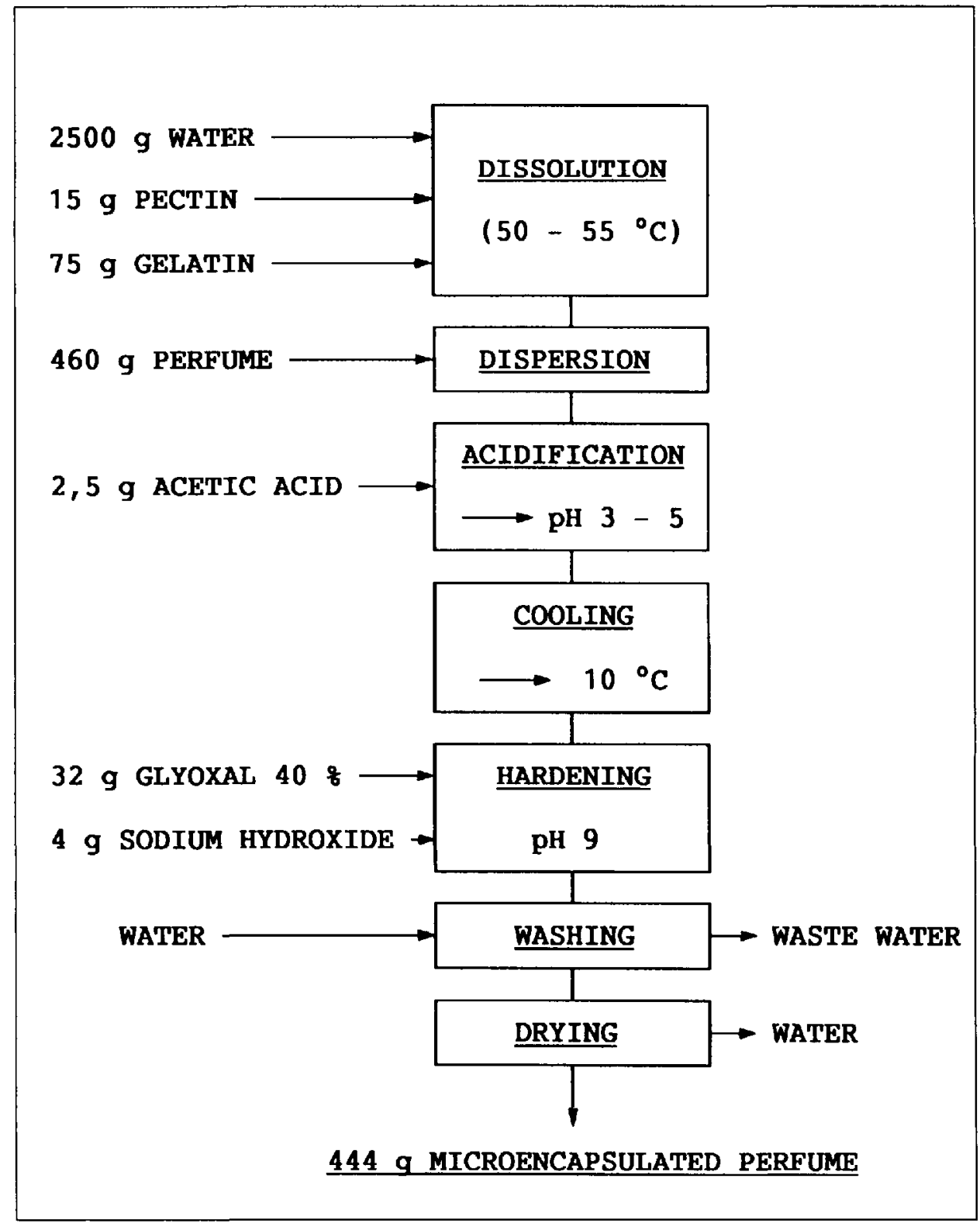

Fig. 1. Process scheme 


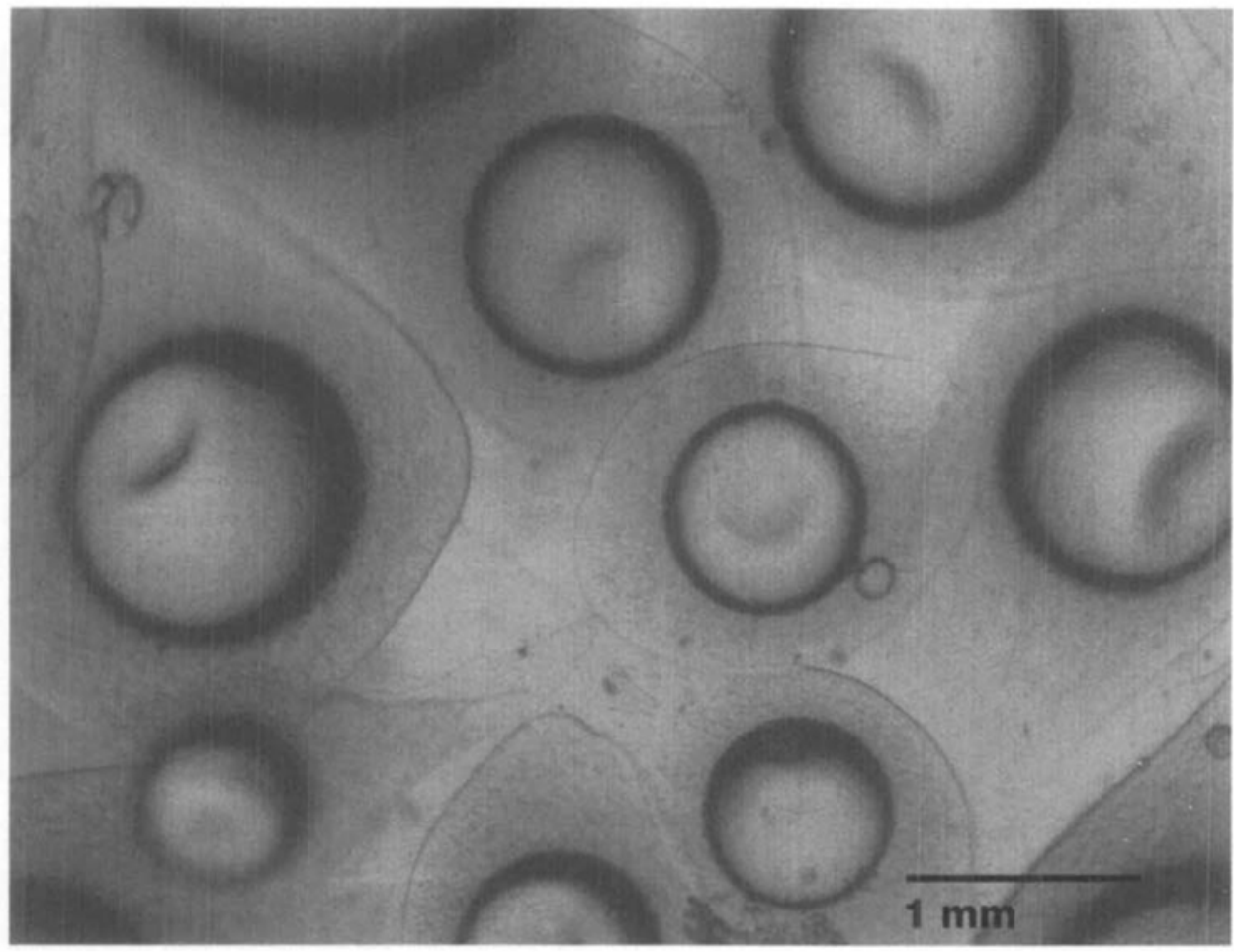

Fig. 2. View of microcapsules

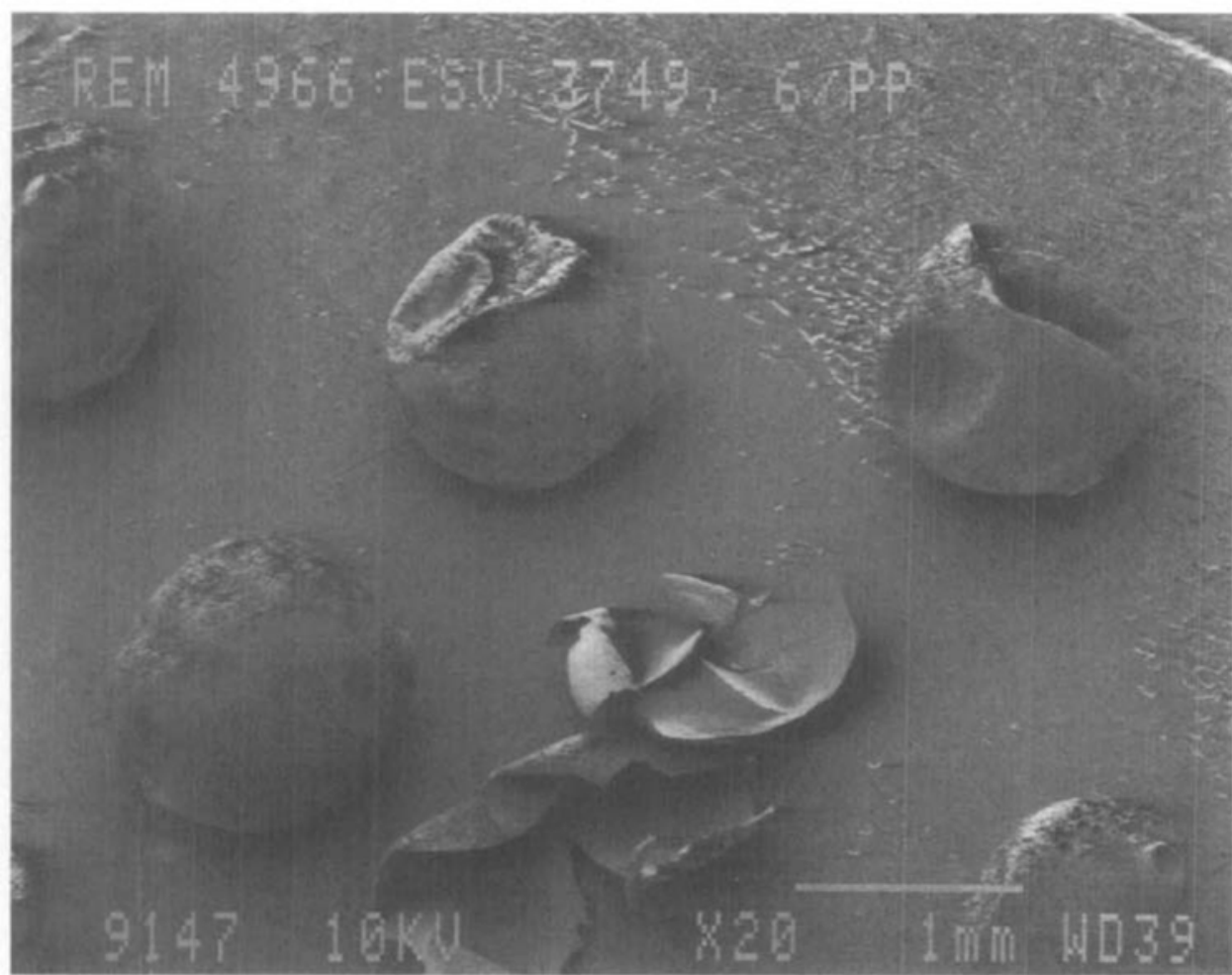

Fig. 3. View of microcapsules taken by a scanning electron microscope

ing microcapsules. The microcapsules were hard ened with $32 \mathrm{~g}$ of $40 \%$ glyoxal under alkaline conditions. After washing and drying, the originally liquid perfume was obtained as free flowing granules. $460 \mathrm{~g}$ of perfume yielded $444 \mathrm{~g}$ of microcapsules having a size from 0.5 to $1.0 \mathrm{~mm}$.

\section{Results}

Fig. 2 shows a view of the microcapsule suspension. A microscope from Olympus, model $B H-2$, was used to take the Polaroid picture. One can clearly dis- middle) to demonstrate the capsule character of the particles obtained.

Although such a thin shell is sufficient to change a liquid perfume to free flowing granules the wall is not completely impermeable and some perfume is lost over the years. Evaporation is faster at higher temperatures. At $60^{\circ}, 5 \%$ of the encapsulated perfume was lost during one month. This compares favorably with microcapsule walls made from gelatin and gum arabic where the loss was $55 \%$ for one month at the same temperature.

\section{Discussion}

Complex coacervation is a suitable technique for encapsulating materials with small solubility in water. Even complex mixtures such as perfumes are easily encapsulated. The same wall materials known to be capable of forming a complex coacervate with gelatin may be used. Always, a liquid perfume is converted to a free flowing powder. However, as far as the performance of the microcapsules is concerned, the wall material has a big influence. For the same wall material-to-perfume weight ratio, much tighter microcapsules are obtained when gelatin is used in combination with pectin instead of gum arabic as was shown in this paper.

\section{Received: January 28, 1992}

[1] R. E. Sparks, 'Microencapsulation, KirkOtmer: Encyclopedia of Chemical Technology', Ed. M. Grayson, 3rd edn., John Wiley \& Sons, New York-Chichester-BrisbaneToronto, 1981, Vol. 15, p. 470-493.

[2] M. H. Gutcho, 'Microcapsules and Microencapsulation Techniques', Noyes Data Corporation, Park Ridge, 1976.

[3] Controlled Delivery in Consumer Products, First Workshop and Exhibition, Cincinnati, Ohio, USA, Oct. 18-19, 1990, Controlled Release Soc. Inc., 16 Nottingham Drive, Lincolnshire, IL 60069, USA.

[4] G. J. De Leeuw, 'Coacervation, micro-encapsulage et produits micro-encapsulés', Parfumerie, Cosmétique, Savons 1970, 13,358.

[5] Y. Nakagawa, T. Kamura, H. Arai, M. Takizawa, S. Konishi, Solid Soap containing Dispersed Microcapsules, Ger. Offen. 3621458, 08.01.1987.

[6] D. W. Michael, Powdered Abrasive Cleansers with Encapsulated Perfume, US 4961871, 09.10.1990. 